\title{
Plasmablastic lymphoma mimicking orbital cellulitis
}

\author{
Richard Barkhuysen - Matthias A. W. Merkx • \\ Willem L. J. Weijs • Niek L. Gerlach • Stefaan J. Bergé
}

Published online: 3 July 2008

(C) The Author(s) 2008

\begin{abstract}
Introduction Orbital cellulitis is an uncommon, potentially devastating condition that, when not promptly and adequately treated, can lead to serious sequelae. The presenting clinical signs are proptosis, swelling, ophthalmoplegia, pain and redness of the peri-orbital tissues. A number of cases have been reported in which these symptoms have been mistakenly interpreted as being secondary to an orbital infection whilst, in fact, other pathology was present. Discussion We add another case in which, on clinical grounds and after radiological assessment and laboratory tests, a working diagnosis of orbital cellulitis of the left eye was made. It was only after histopathological analysis of a soft tissue specimen from the maxillary sinus that a diagnosis of an AIDS-related plasmablastic lymphoma was made. The patient was referred to the department of haematology where chemotherapeutic treatment for the lymphoma and the HIV infection was started. This case report adds another differential diagnosis of orbital cellulitis to the existing literature.
\end{abstract}

Keywords Orbital cellulitis · Plasmablastic lymphoma .

Orbital apex syndrome $\cdot$ Maxillofacial $\cdot$ AIDS

\section{Introduction}

Orbital cellulitis presents with proptosis, swelling, ophthalmoplegia and redness of the surrounding tissues. Since

R. Barkhuysen $(\bowtie) \cdot$ M. A. W. Merkx • W. L. J. Weijs •

N. L. Gerlach · S. J. Bergé

Department of Oral and Maxillofacial Surgery 590,

Radboud University Nijmegen Medical Centre,

P.O. Box 9101, 6500 HB Nijmegen, The Netherlands

e-mail: R.Barkhuysen@mka.umcn.nl these symptoms can also be a manifestation of conditions involving the orbital apex, superior orbital fissure or cavernous sinus, clinicians should take these rather exceptional causes into consideration. A patient is presented in whom initially a diagnosis of orbital cellulitis was made, but that later proved to be a giant plasmablastic lymphoma.

\section{Case}

A 50-year-old female was referred by the department of ophthalmology with a 3-day history of acute onset progressive proptosis, visual loss and ophthalmoplegia of the left eye (Fig. 1). Her medical history was non-contributory.

On physical examination, the patient proved to have a high-grade fever $\left(39.7^{\circ} \mathrm{C}\right)$. There was ophthalmoplegia, inferior displacement and proptosis of the left globe (Hertel [1]; left $26 \mathrm{~mm}$, right $16 \mathrm{~mm}$ ). Visual acuity was decreased. Pupils were isocore and indirect pupillary reflexes were normal. Direct pupillary reflex of the left eye was almost absent. There was anaesthesia of the left maxillary and ophthalmic branches $\left(V_{1}\right.$ and $\left.V_{2}\right)$ of the trigeminal nerve. We also found intra-orally multiple foci in the upper as well as in the lower jaw and a painful swelling of the upper left gingivobuccal sulcus. Leucocyte count was normal but Creactive protein and erythrocyte sedimentation rate were markedly elevated.

Computed tomography (CT) showed an abscess in the left infratemporal fossa with extension of inflammatory tissue to the posterior cranial fossa, temporal fossa, maxillary sinus and orbit. There was also destruction of the skull base. Dental panoramic radiography revealed multiple periapical radiolucencies and caries.

On the basis of these findings, a working diagnosis of an abscess in the left infratemporal fossa with extension to the 


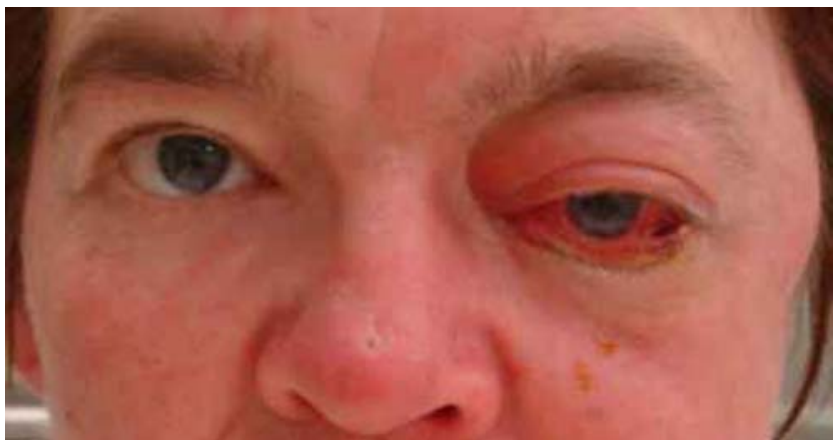

Fig. 1 Proptosis, peri-orbital swelling, redness, hypoglobus and chemosis of the left eye as well as a temporal mass on the left side upon presentation

left orbit causing orbital cellulitis and destruction of the skull base was made. In the differential diagnosis, other possibilities were also taken into consideration, such as a complicating unilateral cavernous sinus thrombosis, osteomyelitis, an underlying malignancy or a rhino-orbitocerebral mucormycosis.

The patient was immediately transferred to the operating ward with the intention to surgically drain a possible abscess as well as to collect material for further histopathological and microbiological investigations. All teeth were extracted. Peroperatively, no pus was encountered. The left maxillary sinus walls were found to be destroyed and the sinus was filled with pathological tissue. Clindamycin (600 mg q 8 h) was started intravenously. There was a favourable response to this as fever resolved, redness of the periorbita disappeared and swelling and proptosis diminished. Infectious laboratory parameters also quickly normalised. Ophthalmoplegia, sensory disturbance of $V_{1}$ and $\mathrm{V}_{2}$ and visual acuity did not improve (Fig. 2 and 3).

Histopathological investigation revealed a plasmablastic lymphoma. Because this disease is AIDS-related, an HIV test was conducted, which proved to be positive. Postoperatively, magnetic resonance imaging (MRI) was made to exclude cavernous sinus thrombosis and to better assess the extension of the diagnosed pathology. Multiple masses

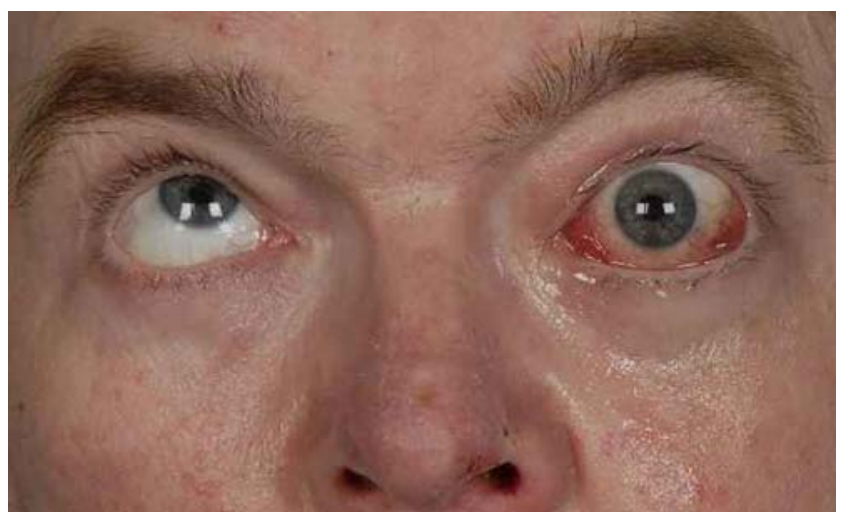

Fig. 2 Ophthalmoplegia on upward gaze

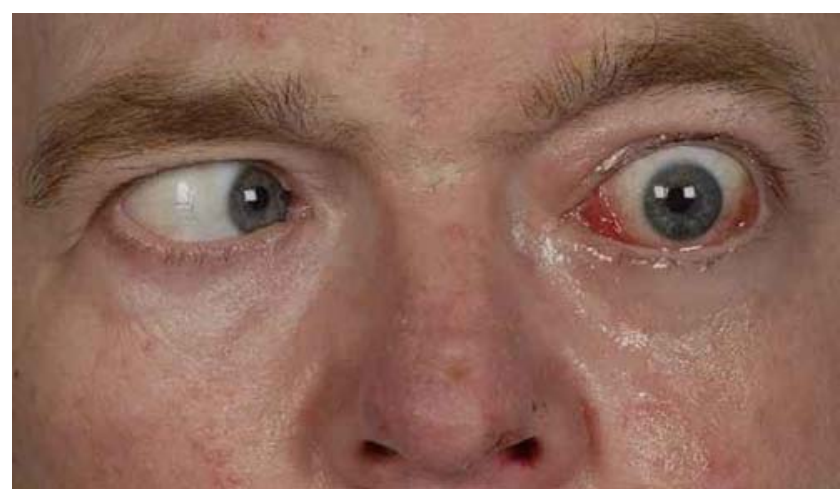

Fig. 3 Ophthalmoplegia on left gaze

were seen (Fig. 4). There was encroachment upon the cavernous sinus and infiltration into the orbit, but no signs of cavernous sinus thrombosis.

The patient was sent to the haematology department where, after dissemination investigation, 18 courses of rituximab, cyclophosphamide, doxorubicin, vincristine and prednisolone chemotherapy (R-CHOP) were given and highly active antiretroviral therapy consisting of emtricitabine, ritonavir, tenofovir and atazanavir was started. She also received 18 sessions of intrathecal chemotherapy (methotrexate alternated with cytarabine/DAF). Because of partial remission after eight courses of R-CHOP, she also received radiotherapy to the skull. A repeated CT scan showed regression of the lymphoma and re-ossification at the skull base.

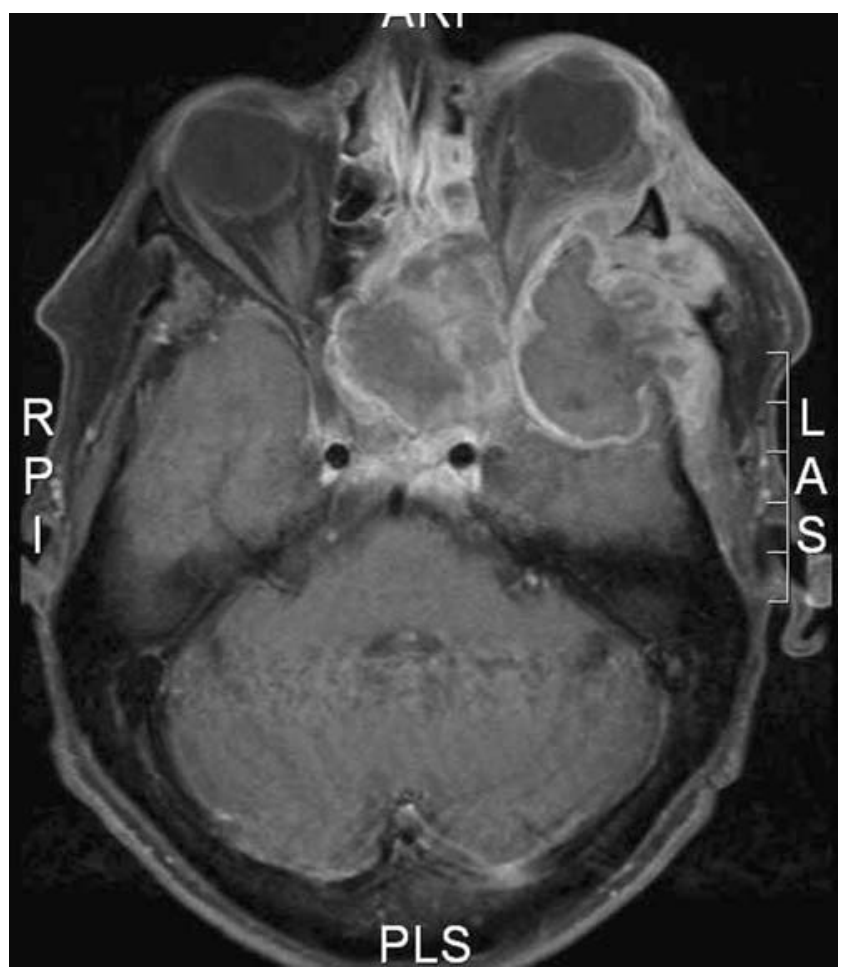

Fig. 4 Axial magnetic resonance image showing huge plasmablastic lymphoma infiltrating the left orbit 


\section{Discussion}

Orbital cellulitis is an infection of the orbital soft tissues posterior to the orbital septum that presents with rapid progressive ophthalmoplegia, proptosis, swelling of the eye, chemosis, decrease in visual acuity, orbital pain and redness of the eyelid [2].

In the majority of patients, it occurs as a direct extension of infection of the paranasal sinuses, but haematogenous spread of bacteria or direct inoculation by trauma or surgery is also possible [3-5]. Sometimes, a dental focus is the underlying cause [6].

The clinical signs, laboratory abnormalities and the presence of a potential dental focus in this patient were suggestive of an infection, i.e. orbital cellulitis. The orbital apex, cavernous sinus and superior orbital fissure are contiguous structures, which may be affected by a number of conditions (inflammatory, infectious, neoplastic, iatrogenic/trauma, vascular) [7].

Depending on the cranial nerves involved and anatomic areas affected, different syndromes can be described:

1. superior orbital fissure syndrome (cranial nerves III, IV, $\left.\mathrm{V}_{1}, \mathrm{VI}\right)$,

2. orbital apex syndrome (cranial nerves II, III, IV, $\mathrm{V}_{1}$, VI),

3. cavernous sinus syndrome (cranial nerves III, IV, $\mathrm{V}_{1}$, $\left.\mathrm{V}_{2}, \mathrm{VI}\right)$.

This classification is only of theoretical importance since the close relation between the mentioned anatomical areas causes a rapid and significant clinical overlap in the presentation of pathological entities.

In light of the symptoms of the described patient (involvement of cranial nerves $\mathrm{V}_{1}$ and $\mathrm{V}_{2}$ ), pathology affecting the cavernous sinus requires special attention as cavernous sinus thrombosis can occur secondary to orbital cellulitis when infection passes posteriorly through the ophthalmic veins. It can begin unilaterally, but will soon progress to become bilateral, and the patient's state of consciousness is often impaired. MRI scanning can and did exclude this condition [8]. The osseous destruction seen on the CT scan led us to suppose the presence of an aggressive or long-standing infectious condition (e.g. mucormycosis) or malignancy (e.g. lymphoma). This fact was not completely appreciated at first due to the patient's acute presentation. Rhino-orbito-cerebral mucormycosis is an aggressive fungal infection that occurs in immune-suppressed patients and presents with proptosis, visual loss and ophthalmoplegia among other symptoms [9]. On CT scanning, bony destruction is often encountered and a definite diagnosis can be made on histopathology [10].

Orbital cellulitis as the primary clinical appearance of a lymphoma has been reported in the literature before [11, 12].
The diagnosis of a plasmablastic lymphoma with regard to this is, however, unique. In this case, immunopathology revealed expression of CD45 and a weak expression of CD138 and CD79a. There was no expression of CD56, CD30, CD3, CD20 and CD10. Epstein-Barr virus-encoded RNA in situ hybridisation was positive. Plasmablastic lymphoma is a rare, AIDS-related, non-Hodgkin lymphoma with a noticeable predilection for the oral cavity [13]. This entity was first described by Delecluse et al. [14]. It carries a dismal prognosis with an average survival time of 6 months after diagnosis. At 13 months after diagnosis, our patient is still alive.

In retrospect, although there was an infectious component, the presence of the acute orbital symptoms in this case were most likely secondary to the lymphoma that was widespread to the skull base and orbit.

Yeh et al. presented a diagnostic algorithm for patients with findings consistent with an orbital apex syndrome [7]. Imaging should be performed in all cases, and MRI scanning is considered being superior to other imaging modalities. When inflammatory or infectious disease is suspected, proper laboratory tests should be conducted as well. Obtaining material for histopathological analysis may be required to arrive at a definite diagnosis.

On the basis of this case report, plasmablastic lymphoma may be added to the list of differential diagnoses of orbital cellulitis.

Open Access This article is distributed under the terms of the Creative Commons Attribution Noncommercial License which permits any noncommercial use, distribution, and reproduction in any medium, provided the original author(s) and source are credited.

\section{References}

1. Mourits MP, Lombardo SHC, van der Sluijs FA, Fenton S (2004) Reliability of exophthalmus measurement and the exophthalmometry value distribution in a healthy Dutch population and in Graves' patients. An exploratory study. Orbit 23:161-168

2. Chaudhry IA, Shamsi FA, Elzaridi E et al (2007) Outcome of treated orbital cellulitis in a tertiary eye care center in the middle east. Ophthalmology 114:345-354

3. Simon GJB, Bush S, Selva D, McNab AA (2005) Orbital cellulitis: a rare complication after orbital blowout fracture. Ophthalmology 112:2030-2034

4. Dhariwal DK, Kittur MA, Farrier JN, Sugar AW, Aird DW, Laws DE (2003) Post-traumatic orbital cellulitis. Br J Oral Maxillofac Surg 41:21-28

5. Tovilla-Canales JL, Nava A, Tovilla y Pomar JL (2001) Orbital and periorbital infections. Curr Opin Ophthalmol 12:335341

6. Kim IK, Kim JR, Jang KS, Moon YS, Park SW (2007) Orbital abscess from an odontogenic infection. Oral Surg Oral Med Oral Pathol Radiol Endo 103:e1-e6 
7. Yeh S, Foroozan R (2004) Orbital apex syndrome. Curr Opin Ophthalmol 15:490-498

8. Igarashi H, Igarashi S, Fujio N, Fukui K, Yoshida A (1995) Magnetic resonance imaging in the early diagnosis of cavernous sinus thrombosis. Ophthalmologica 209:292-296

9. Harrill WC, Stewart MG, Lee AG, Cernoch P (2007) Chronic rhinocerebral mucormycosis. Laryngoscope 106:1292-1297

10. Nithyanandam S, Jacob MS, Batty RR, Thomas RK, Correa MA, D'Souza O (2003) Rhino-orbito-cerebral mucormycosis. A retrospective analysis of clinical features and treatment outcomes. Indian J Ophthalmol 51:231-236
11. Nakajima A, Abe T, Takagi T et al (1996) Two cases of malignant lymphoma complicated by hemophagocytosis resembling orbital cellulitis. Jpn J Ophthalmol 41:186-191

12. Salam A, Saldana M, Zaman N (2005) Orbital cellulitis or lymphoma? A diagnostic challenge. J Laryngol Otol 119:740-742

13. Flaitz CM, Nichols CM, Walling DM, Hicks MJ (2002) Plasmablastic lymphoma: an HIV-associated entity with primary oral manifestations. Oral Oncol 38:96-102

14. Delecluse HJ, Dallenbach F, Hummel M et al (1997) Plasmablastic lymphomas of the oral cavity: a new entity associated with the human immunodeficiency virus infection. Blood 89:1413-1420 